\section{Polyhedral Oligomeric Silsesquioxane-Reinforced Photopolymers Demonstrate High Sensitivity for Holographic Data Storage}

Holographic data storage is a promising new method for recording digital information. This is because holography allows pages of data to be written at once, while storing multiple pages of data in a given volume. Photopolymers are attractive materials for use as holographic recording media due to their high sensitivity and low cost. However, a critical drawback of photopolymers is the volume shrinkage which occurs during polymerization. Now J.-K. Park and co-workers from Korea Advanced Institute of Science and Technology (KAIST) have shown that incorporating multifunctional molecular silicate "cages" into the polymer matrix of the holographic photopolymer reduces volume shrinkage while maintaining high photosensitivity.
As reported recently in Optics Letters, to minimize volume shrinkage, the researchers created an organic-inorganic hybrid material using methacrylate-functionalized polyhedral oligomeric silsesquioxane (POSS) as a co-monomer. The polymer matrix consisted of PMMA with dibutylphthalate plasticizer, $n$-vinylcarbazole as a second co-monomer, and Irgacure 784 as the photoinitiator. Three compositions were tested with varying amounts of POSS: 0,1 , and $2 \mathrm{wt} \%$.

The researchers showed higher diffraction efficiency with systems that contained POSS. A maximum diffraction efficiency of $80 \%$ was achieved using $1 \mathrm{wt} \%$ POSS, compared to $\sim 30 \%$ without POSS. Additionally, the diffraction efficiency increased more rapidly with higher concentrations of POSS. As a result, the photosensitivity more than doubled when $1 \mathrm{wt} \%$ POSS was added to the system. The researchers attribute the rapid growth in diffraction efficiency to the multiple vinyl groups provided by each POSS molecule. By increasing the polymerization rate, a larger concentration gradient is created for the $n$-vinylcarbazole monomer. This drives the diffusion of the monomer into the illuminated region and increases the diffraction efficiency. The dimensional stability of the photopolymer system was determined using Bragg angle detuning. From this experiment, the volume shrinkage was calculated to be $2.0 \%$ for systems containing $1 \mathrm{wt} \%$ POSS, compared to $3.0 \%$ for systems without POSS. The researchers conclude that using the multifunctional POSS molecule as a co-monomer strengthens the polymer matrix of the holographic photopolymer and reduces volume shrinkage while maintaining high diffraction efficiency and photosensitivity.

SCOTT COOPER

\section{Metal Organic Frameworks Achieve $\mathrm{CO}_{2}$-Specific Adsorption at Low Pressures}

Metal-organic frameworks (MOFs) have emerged as an important class of materials due to their porous nature and relative ease of design. Their low density $\left(0.20-1.00 \mathrm{~g} / \mathrm{cm}^{3}\right)$ and high surface area (500-4500 $\mathrm{m}^{2} / \mathrm{g}$ ) have brought MOFs into the forefront of gas storage and separation research. To date, a significant portion of relevant publications has focused on large-pore MOFs due to their ability to store higher volumes of gas. However, high pressures are usually needed in order for large-pore MOFs to adsorb extreme amounts of gases. Additionally, gas selectivity would be expected to decrease with increasing pore size. In general, gas sorption can be controlled by the MOF pore structures and the functionality present within the pores.

As recently reported in Chemical Communications (Issue 35, DOI: 10.1039/ b911481e; p. 5230), R. Vaidhyanathan, S.S. Iremonger, K.W. Dawson, and G.K.H. Shimizu of the University of Calgary have achieved specific $\mathrm{CO}_{2}$ adsorption at low pressure. Starting with commercially available materials-zinc carbonate, oxalic acid, 3-amino-1,2,4triazole-highly crystalline MOFs with small pores $(3.5 \AA \times 4.0 \AA$ and $3.9 \AA \times 2.1 \AA$ ) were synthesized. By incorporating amine-containing molecules, the pores of the final product are functionalized with free amines, which are purported to interact with $\mathrm{CO}_{2}$. After activating the MOFs to remove all solvent, the adsorption of several gases was studied at various temperatures. Particularly of note, $\mathrm{CO}_{2}$ sorption was as high as $4.35 \mathrm{mmol} / \mathrm{g}$ at $273 \mathrm{~K}$ with partial pressures as low as 1.2 bar. The experimental adsorption and desorption were completely reversible for the eight trials that were repeated. The adsorption of $\mathrm{N}_{2}, \mathrm{H}_{2}$, and Ar were also studied, with only minimal uptake of these gases observed. The selectivity of the designed MOF is attributed to a combination of the small pores and the extensive host-guest interactions due to the presence of the free amines.

Vaidhyanathan and collaborators said this MOF may potentially be useful for removing trace amounts of $\mathrm{CO}_{2}$ from recyclable gases due to its selectivity at low partial pressures. Future research aims to characterize related materials as well as the physical nature of the adsorbed $\mathrm{CO}_{2}$ molecules.

DEVIN G. BARRETT

\section{Sulfur- and Coking-Tolerant Material Could Expand Applications for SOFCs}

Solid-oxide fuel cells (SOFCs) use an electrochemical process to produce electricity by oxidizing a fuel. The ceramic electrolyte they use is yttria-stabilized zirconia (YSZ). The main drawbacks to SOFCs are their susceptibility to contamination from sulfur, deactivation from carbon build-up, and limited conductivity at low temperatures. Now M. Liu and colleagues at the Georgia Institute of Technology have developed a mixed ion conductor,
$\mathrm{BaZr}_{0.1} \mathrm{Ce}_{0.7} \mathrm{Y}_{0.2-x} \mathrm{Yb}_{x} \mathrm{O}_{3-\delta}(\mathrm{BZCYYb})$ that may address these problems.

Fuels used in SOFCs, such as natural gas or propane, must be purified to remove sulfur, which increases their cost. Water in the form of steam must also be supplied to a reformer that converts hydrocarbons to hydrogen and carbon monoxide before being fed to the fuel cells, adding complexity to the overall system and reducing energy efficiency. And the high-temperature operation means the cells must be fabricated from costly exotic materials, which keeps SOFCs too expen- sive for many applications.

As reported in the October 2 issue of Science (DOI: 10.1126/science.1174811; p. 126), the new material developed at Georgia Tech tolerates hydrogen sulfide in concentrations as high as $50 \mathrm{ppm}$, does not accumulate carbon, and can operate efficiently at temperatures as low as $500^{\circ} \mathrm{C}$.

The material could be used in a variety of ways: as a coating on the traditional NiYSZ anode, as a replacement for the YSZ in the anode, and as a replacement for the entire YSZ electrolyte system. Liu believes the first two options are more viable. 